\title{
ROCK1 and ROCK2 Are Down-regulated in Aggressive and Advanced Skin Melanomas - A Clinicopathological Perspective
}

\author{
MACIEJ KACZOROWSKI ${ }^{1}$, PRZEMYSŁAW BIECEK ${ }^{2}$, PIOTR DONIZY ${ }^{1}$, \\ MAŁGORZATA PIENIĄŻEK ${ }^{3}$, RAFAŁ MATKOWSKI ${ }^{4,5}$ and AGNIESZKA HAŁOŃ 1,5 \\ ${ }^{1}$ Department of Pathomorphology and Oncological Cytology, Wroclaw Medical University, Wroclaw, Poland; \\ ${ }^{2}$ Faculty of Mathematics and Information Science, Warsaw University of Technology, Warsaw, Poland; \\ ${ }^{3}$ Department of Clinical Oncology, Tadeusz Koszarowski Regional Oncology Centre, Opole, Poland; \\ ${ }^{4}$ Division of Surgical Oncology, Department of Oncology, Wroclaw Medical University, Wroclaw, Poland; \\ ${ }^{5}$ Wroclaw Comprehensive Cancer Center, Wroclaw, Poland
}

\begin{abstract}
Background: RhoA and its downstream effectors Rho-associated coiled-coil kinases (ROCK) 1 and 2 are central controllers of cytoskeleton dynamics, and therefore influence cell shape, adhesion and migration. Since modulation of these processes holds promise for an effective anticancer strategy, effects of ROCK inhibition have been evaluated in a number of malignancies. Materials and Methods: Using immunohistochemistry, ROCK1 and ROCK2 expression was semi-quantitatively assessed in 129 patientderived primary melanomas. Results: There was a striking predilection for low melanocytic expression of both kinases in thick, ulcerated and mitogenic tumors, as well as in nodular histological type. ROCK1 and -2 expression in tumor-infiltrating lymphocytes (TILs) was preferentially down-regulated in advanced and aggressive tumors. Moreover, diminished ROCK2 reactivity in melanoma cells and TILs was associated with shorter melanoma-specific and recurrence-free survival. Conclusion: This is the first analysis of ROCK1 and -2 protein expression in clinical melanoma samples and the results indicated the suppression of ROCK signaling in melanocytes of aggressive and late-stage tumors. Functional models that more accurately represent the clinical setting are necessary to dissect the role of ROCK1 and -2 in melanoma. Additionally, our study indicates that ROCK
\end{abstract}

This article is freely accessible online.

Correspondence to: Maciej Kaczorowski, MD, Department of Pathomorphology and Oncological Cytology, Wroclaw Medical University, Borowska 213, 50-556 Wroclaw, Poland. Tel: +48 717343960, Fax: +48 717343968, e-mail: maciejdkaczorowski@ gmail.com ORCID: 0000-0002-7787-9952

Key Words: ROCK1, ROCK2, melanoma, tumor-infiltrating lymphocytes, prognosis, Kaplan-Meier analysis. activity in TILs may be involved in the pathogenesis of cancer, and thus merits further investigations.

Cellular motility and invasiveness are key determinants of cancer progression and metastatic spread. Since chances for a durable remission are usually significantly diminished by the development of tumor metastases, mechanisms that regulate this process have been vigorously investigated. Cognition of biochemical agents accountable for cancer dissemination is even more alluring in the current era of novel, molecular-based therapies that may specifically target dysregulated pathways.

A number of studies implicated the family of Rho GTPases and their immediate downstream effectors Rho-associated coiled-coil kinases (ROCKs) as central controllers of important cellular processes, such as cell shape, adhesion or migration (1). Mammalian ROCK proteins consist of two highly homologous isoforms, ROCK1 and ROCK2 (1). Their canonical activity involves promoting actomyosin contractility through phosphorylation of myosin light chain and stabilizing actin filaments through activation of LIM kinase (1). This influence on cytoskeleton dynamics endows ROCKs with a regulatory function over several hallmark features of cancer, e.g. invasion, cell division and remodeling of the extracellular matrix (2). Consequently, the effects of ROCK inhibition have been extensively studied in various tumor types. Employment of gene knockdown techniques and protein inhibitors, such as fasudil or Y27632, effectively reduced proliferation, invasion and formation of metastases in a majority of investigations on melanoma, breast, pancreatic, hepatic, ovarian and other types of cancer (3-8). Moreover, depletion of ROCKs reduced cell viability and induced apoptosis in bladder and hematological malignancies, as well as in melanoma (9-11). Conversely, several other groups demonstrated deleterious effects of ROCK inhibition. Treatment with ROCK inhibitor Y27632 led to increased migration and mitotic activity in breast, colon and pancreatic cancer cell cultures (12-15). Others showed that 
inactivation of ROCK signaling promoted cell survival and attenuated drug-induced apoptosis in leukemia, neuroblastoma and ovarian carcinoma (16-18), as well as increased cancer cell stemness $(19,20)$. These seemingly contradictory findings may be at least partly attributed to the choice of different cell lines in various studies. In melanoma, substitution of B16F1 culture with UACC257 or UACC62 cells resulted in opposite observations in proliferation and migration assays - ROCK inhibition impaired melanoma cell growth and motility in the first cell line and promoted these functions in the latter two $(11,21,22)$. This illustrates an important caveat to past functional studies - they do not account for the considerable molecular heterogeneity of naturally occurring melanomas. Therefore, their conclusions regarding pro- or antitumor activities of ROCKs may not be easily generalizable to a broad clinical spectrum of skin melanomas.

To date, expression profiles of ROCK 1 and ROCK 2 proteins have not been characterized in clinical melanoma samples. With this aim, we investigated immunoreactivity of ROCK1 and ROCK2 in the neoplastic compartment of primary melanomas and explored associations between the observed protein expression and other pathological and clinical characteristics. Since the RhoA-ROCK axis plays a regulatory role in immune responses $(23,24)$, we additionally evaluated expression of ROCK1 and ROCK2 in tumor-infiltrating lymphocytes (TILs).

\section{Materials and Methods}

Patient samples. Tissue samples were obtained from 129 patients diagnosed with and treated for skin melanoma between 2005 and 2010 in the Regional Oncology Centre in Opole, Poland. Inclusion criteria were: Availability of paraffin blocks and corresponding histopathology slides, and availability of archival medical documentation, including disease staging and original pathology reports. Data regarding applied medical procedures and patient survival were obtained from medical records of the Regional Oncology Centre in Opole and the Civil Register Office. The study was approved by the Bioethics Committee of Wroclaw Medical University (approval no.: KB-574/2017) which waived the necessity for informed consent and was carried out in accordance with the Declaration of Helsinki.

The patients' treatment was concordant with the prevailing guidelines. Following the histopathological diagnosis of cutaneous melanoma, the primary lesions were excised with a margin of 5, 10 or $20 \mathrm{~mm}$ depending on tumor thickness and localization. Sentinel lymph node biopsies were performed in cases with no clinical evidence of metastases (cNO) and Breslow thickness exceeding 1 $\mathrm{mm}(>\mathrm{pT} 1 \mathrm{a})$. Lymphadenectomies were applied in cases with metastases in the regional lymph nodes (found clinically or by sentinel lymph node biopsy).

Clinicopathological parameters included information about patient age, sex, location of primary tumors, their TNM classification and staging according to the guidelines of American Joint Committee on Cancer (AJCC) (seventh edition) (25), information about disease recurrence and sentinel lymph node biopsy procedures.

Formalin-fixed and paraffin-embedded tissues of primary tumors were used to prepare $4 \mu \mathrm{m}$-thick sections that were subsequently stained by hematoxylin and eosin. All slides were blindly reviewed by two pathologists (MK and PD). Recorded histopathological characteristics included: Breslow thickness, Clark level, histological type, mitotic index (per $1 \mathrm{~mm}^{2}$ ), and the presence of ulceration, microsatellites, lymphovascular invasion and TILs. The last parameter was assessed semi-quantitatively with three possible grades: Absent, non-brisk and brisk.

Immunohistochemistry. Paraffin blocks with tissues of primary melanomas were cut with a microtome to prepare $4 \mu \mathrm{m}$-thick sections which were subsequently mounted on sialinized slides (Agilent DAKO, Santa Clara, CA, USA). The slides then underwent automated dewaxing, rehydration and heat-induced epitope retrieval with EnVision Target Retrieval Solution (Agilent DAKO) in a 30minute incubation at $97^{\circ} \mathrm{C}$ in PT Link Pre-Treatment Module for Tissue Specimens (DAKO). Automated immunohistochemical staining with anti-ROCK1 (rabbit monoclonal, dilution 1:100; Abcam, Cambridge, UK) and anti-ROCK2 (rabbit polyclonal; dilution 1:100; Abcam) was performed in Autostainer Link 48 (DAKO) and Liquid Permanent Red (Agilent DAKO) was utilized as a detection system. Human colorectal adenocarcinoma and human hepatocellular carcinoma tissues were stained as positive controls for ROCK1 and ROCK2 antibodies, respectively. Negative controls were processed using FLEX Rabbit Negative Control, Ready-to-Use (Agilent DAKO) in place of the primary antibody.

Evaluation of immunohistochemistry. Expression of ROCK1 and ROCK2 was evaluated in two compartments, namely melanoma cells and TILs. The semi-quantitative scale of Remmele and Stegner (26) combining two parameters of immunohistochemical reaction, i.e. the percentage of reactive tissue and staining intensity, was modified by the authors and employed as described previously for evaluation of immunoexpression in tumor cells (27). In brief, 0-3 points were given for the intensity of reaction and 0-10 points were given (0\%: 0 points, $1-10 \%$ : 1 point, $11-20 \%$ : 2 points, etc.) for the percentage of reactive neoplastic cells. Multiplication of these two values gave a product ranging from 0 to 30 points named the immunoreactive score (IRS). Expression of ROCK proteins in TILs was graded as absent/weak, medium or strong based on reaction intensity juxtaposed with the staining intensity of positive controls. An Olympus BX51 light microscope (Olympus America, Inc., Melville, NY, USA) was used for the evaluation of slides.

Statistical analysis. Statistical analyses were performed in $\mathrm{R}$ language. Expression of ROCK1 and ROCK2 in cancer cells were transformed into binary variables with the use of maxstat package. In both cases, the cutoff IRS of $\leq 20 v s$. IRS $>20$ was used to differentiate between low and high immunoexpression. Continuous variables, including patient age and Breslow thickness, were characterized with their mean, median, and range values. KaplanMeier curves and corresponding log-rank tests were applied with the survminer package, for analysis of melanoma-specific survival (MSS) and recurrence-free survival (RFS). Relationship of ROCK1 and -2 expression in melanocytes and TILs with continuous variables was assessed by the Wilcoxon two-sample test and Kruskal-Wallis test, respectively. Associations of IRS with binary variables were calculated by Fisher's exact test and the relationships with other categorical variables, as well as associations between ROCK 1 and -2 expression in TILs and categorical variables, were analyzed by chi-squared test. Values of $p<0.05$ were accepted as a threshold for statistical significance. 

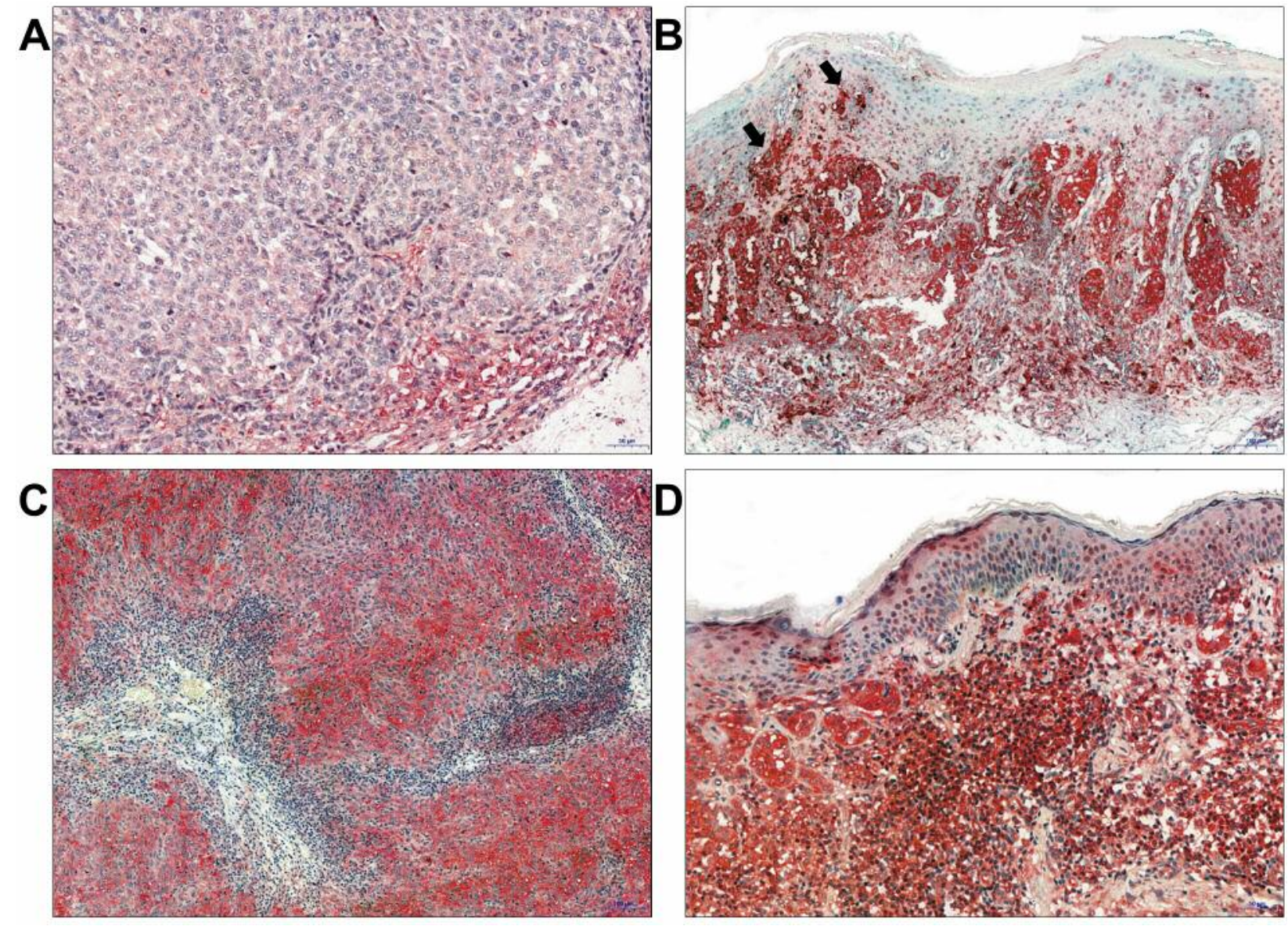

Figure 1. Immunohistochemical visualization of Rho-associated coiled-coil kinase 1 (ROCK1) expression in cutaneous melanomas. A: Weak cytoplasmic staining of melanoma cells with a rim of stronger-stained immune cells on the lower right $(\times 200)$. B: Strong staining in melanocytic nests and foci of pagetoid spread (arrows) $(\times 100)$. C: Neoplastic infiltrate transected by fibrous stroma containing brisk lymphocytic infiltrate with low ROCK1 immunoreactivity (×200). D: Both tumor-infiltrating lymphocytes and melanoma cells highly reactive for ROCK1 (×100).

\section{Results}

Expression of ROCK1 and ROCK2 proteins in skin melanomas. Cytoplasmic immunoreactivity for ROCK1 and ROCK2, ranging from weak and focal to strong and diffuse, was observed in neoplastic compartments of all 129 primary tumors (Figure 1A and B, and Figure 2A and B). Median and mean IRS for ROCK1 were 20 and 19.8, respectively, whereas for IRS ROCK2 these values were 21 and 20.9 , respectively. Slightly over $40 \%$ of melanomas were classified as high ROCK 1 expressors and $46 \%$ as high ROCK2 expressors. IRS for ROCK1 and ROCK2 were moderately correlated (Spearman's $\mathrm{r}=0.6, p<0.0001$; data not shown). In our previous study on skin melanoma, we analyzed expression of RhoA GTPase, a direct controller of ROCK1 and ROCK2 function (28). Interestingly, ROCK1 and ROCK2 immunoreactivity did not correlate with RhoA expression (Figure 3). As regards TILs, at least focal positive staining for
ROCK1 was present in 124 cases, being weak in 51, medium in 45 and strong in 28 tumors (Figure 1C and D). Reaction for ROCK2 was also positive in all evaluated cases: Weak in 28, medium in 72 and strong in 24 melanomas (Figure 2C and D). Five tumors with no apparent lymphocytic infiltrate (TIL grade: Absent) were excluded from this analysis.

Expression of ROCK1 and ROCK2 in melanoma cells and clinicopathological characteristics. ROCK1 expression in our cohort was mostly unrelated to the clinical background but ROCK2 was expressed more strongly in older patients. Melanoma recurred more often in those with tumors with low ROCK2 staining, but there was only a trend for a relation with ROCK1, without clear statistical significance. Melanocytic expression of both ROCK proteins dropped with advancing $\mathrm{pT}$ stage. This relation was particularly evident with regard to ROCK2 staining, with 93\% of high expressors among pT1 tumors and only $20 \%$ among pT4 cases. 


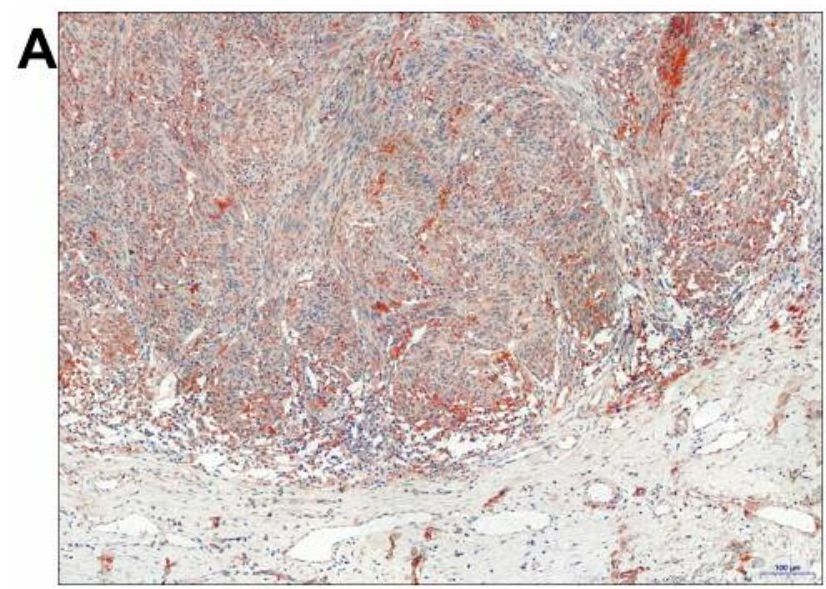

B
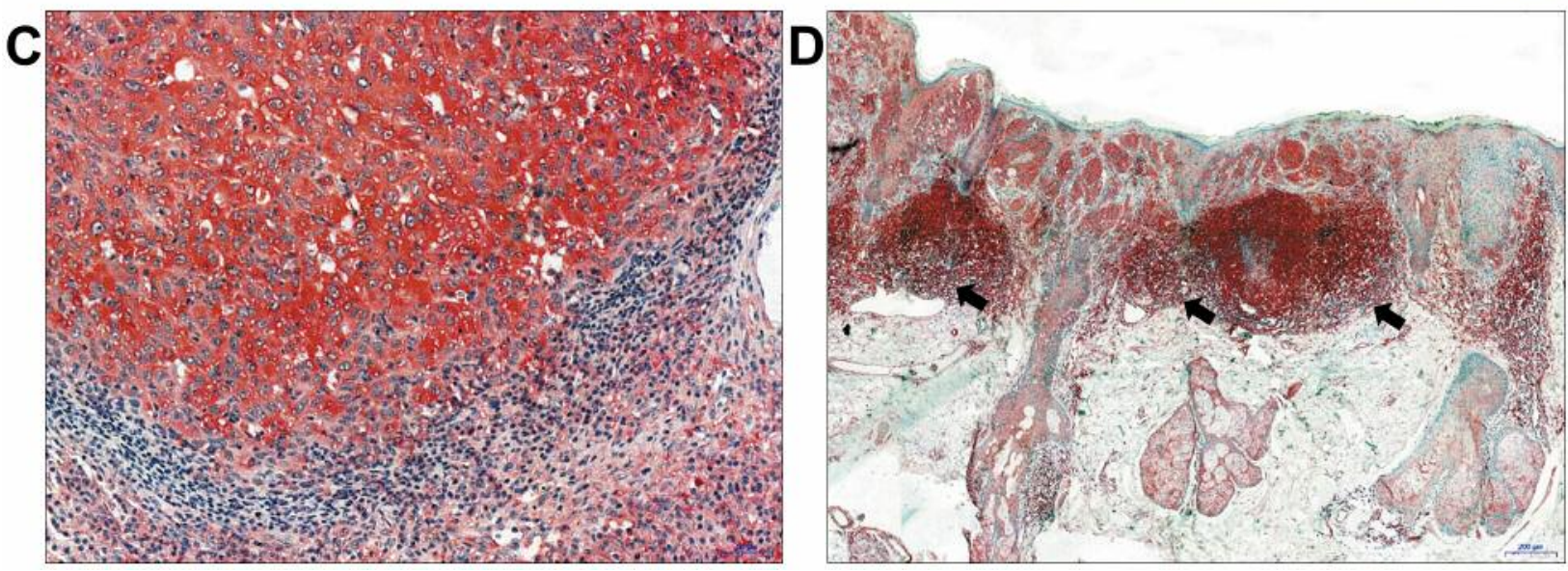

Figure 2. Immunohistochemical visualization of Rho-associated coiled-coil kinase 2 (ROCK2) expression in cutaneous melanomas. A: Base of a nodular melanoma with weak staining of tumor cells $(\times 100)$. B: Melanoma nests strongly positive for ROCK2 (×100). C: Nodule of malignant melanocytes surrounded by a rim of lymphocytes that are weakly positive for ROCK2 (×200). D: Brisk accumulation of tumor-infiltrating lymphocytes (arrows) with intense ROCK2 staining ( $\times 50)$.

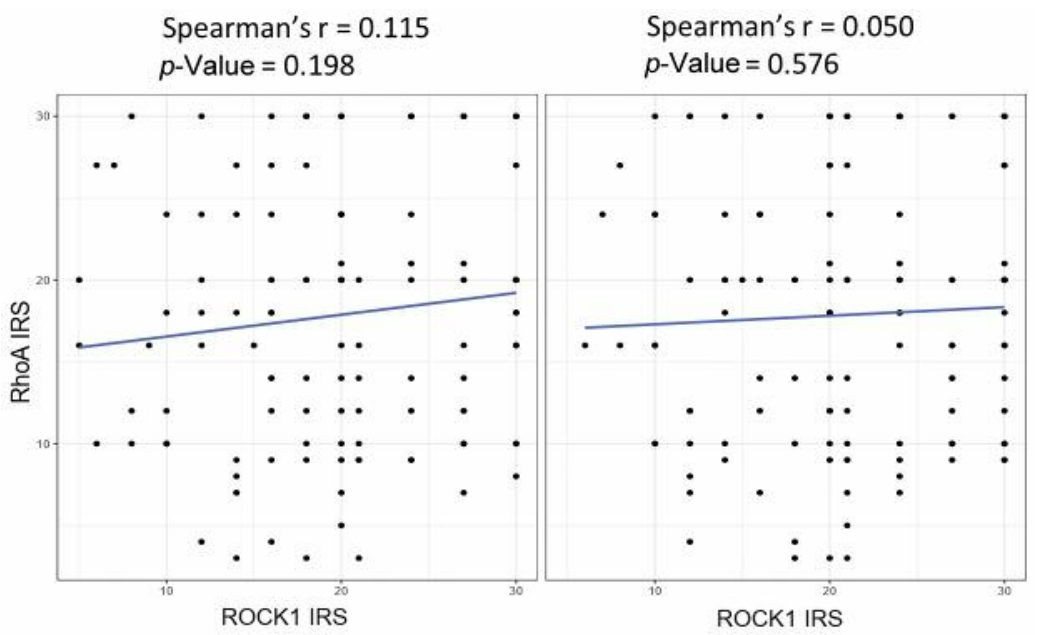

Figure 3. Scatter plots demonstrating characteristics of immunoreactivity for Rho-associated coiled-coil kinase (ROCK) 1 and 2 throughout the study population together with corresponding values of RhoA immunoreactive score (IRS). Dots represent individual tumors and lines represent trend. 
Table I. Clinical characteristics of melanoma patients according to Rho-associated coiled-coil kinase 1 (ROCK1) expression.

\begin{tabular}{|c|c|c|c|c|c|c|c|c|}
\hline \multirow[t]{3}{*}{ Parameter } & \multirow[b]{3}{*}{$\begin{array}{c}\text { Total } \\
(\mathrm{n}=129)\end{array}$} & \multicolumn{6}{|c|}{ ROCK1 immunoreactivity } & \\
\hline & & \multicolumn{3}{|c|}{ Melanoma cells } & \multicolumn{4}{|c|}{ Tumor-infiltrating lymphocytes } \\
\hline & & $\begin{array}{l}\text { Low } \\
(\mathrm{n}=76)\end{array}$ & $\begin{array}{c}\text { High } \\
(\mathrm{n}=532)\end{array}$ & $p$-Value & $\begin{array}{c}\text { Weak } \\
(\mathrm{n}=518)\end{array}$ & $\begin{array}{c}\text { Medium } \\
(\mathrm{n}=45)\end{array}$ & $\begin{array}{l}\text { Strong } \\
(\mathrm{n}=28)\end{array}$ & $p$-Value \\
\hline \multicolumn{9}{|l|}{ Age, years } \\
\hline Mean \pm SD & $62 \pm 15$ & $63 \pm 15$ & $60 \pm 14$ & $0.29^{\mathrm{a}}$ & $64 \pm 15$ & $61 \pm 14$ & $62 \pm 16$ & $0.56^{\mathrm{b}}$ \\
\hline Median & $65(18-87)$ & $67(18-87)$ & $64(25-82)$ & & $69(18-86)$ & $62(24-87)$ & $65(25-81)$ & \\
\hline \multicolumn{9}{|l|}{ Gender, $\mathrm{n}(\%)$} \\
\hline Female & $67(52 \%)$ & $38(50 \%)$ & $29(55 \%)$ & & $26(51 \%)$ & $27(60 \%)$ & $12(43 \%)$ & \\
\hline Male & $62(48 \%)$ & $38(50 \%)$ & $24(45 \%)$ & $0.72^{\mathrm{c}}$ & $25(49 \%)$ & $18(40 \%)$ & $16(57 \%)$ & $0.35^{\mathrm{d}}$ \\
\hline \multicolumn{9}{|c|}{ Primary tumor location, $\mathrm{n}(\%)$} \\
\hline Head/neck & $12(9 \%)$ & $10(13 \%)$ & $2(4 \%)$ & & $5(10 \%)$ & $3(7 \%)$ & $4(14 \%)$ & \\
\hline Extremities & $55(43 \%)$ & $29(38 \%)$ & $26(49 \%)$ & $0.24^{\mathrm{d}}$ & $24(47 \%)$ & $22(49 \%)$ & $7(25 \%)$ & $0.28^{\mathrm{d}}$ \\
\hline Hand/foot & $4(3 \%)$ & $3(4 \%)$ & $1(2 \%)$ & & $1(2 \%)$ & $2(4 \%)$ & $0(0 \%)$ & \\
\hline Trunk & $58(45 \%)$ & $34(45 \%)$ & $24(45 \%)$ & & $21(41 \%)$ & $18(40 \%)$ & $17(61 \%)$ & \\
\hline \multicolumn{9}{|c|}{ AJCC stage, $\mathrm{n}(\%)^{*}$} \\
\hline I & $46(36 \%)$ & $16(21 \%)$ & $30(57 \%)$ & & $12(24 \%)$ & $16(36 \%)$ & $17(61 \%)$ & \\
\hline II & $47(36 \%)$ & $37(49 \%)$ & $10(19 \%)$ & $0.0002^{d}$ & $23(45 \%)$ & $15(33 \%)$ & $8(29 \%)$ & $0.049^{d}$ \\
\hline III & $24(19 \%)$ & $16(21 \%)$ & $8(15 \%)$ & & $12(24 \%)$ & $8(18 \%)$ & $2(7 \%)$ & \\
\hline IV & $12(9 \%)$ & $7(9 \%)$ & $5(9 \%)$ & & $4(8 \%)$ & $6(13 \%)$ & $1(4 \%)$ & \\
\hline \multicolumn{9}{|c|}{ Primary tumor (pT), n (\%) } \\
\hline pT1 & $30(23 \%)$ & $8(11 \%)$ & $22(42 \%)$ & & $7(14 \%)$ & $9(20 \%)$ & $13(46 \%)$ & \\
\hline pT2 & $22(17 \%)$ & $10(13 \%)$ & $12(23 \%)$ & $<0.0001^{a}$ & $6(12 \%)$ & $11(24 \%)$ & $5(18 \%)$ & $0.0014^{b}$ \\
\hline pT3 & $32(25 \%)$ & $22(29 \%)$ & $10(19 \%)$ & & $10(20 \%)$ & $14(31 \%)$ & $6(21 \%)$ & \\
\hline pT4 & $45(35 \%)$ & $36(47 \%)$ & $9(17 \%)$ & & $28(55 \%)$ & $11(24 \%)$ & $4(14 \%)$ & \\
\hline \multicolumn{9}{|c|}{ Regional lymph node status (pN), n (\%) } \\
\hline $\mathrm{pN}-$ & $98(76 \%)$ & $55(72 \%)$ & $43(81 \%)$ & & $35(69 \%)$ & $34(76 \%)$ & $26(93 \%)$ & \\
\hline $\mathrm{pN}+$ & $31(24 \%)$ & $21(28 \%)$ & $10(19 \%)$ & $0.30^{\mathrm{c}}$ & $16(31 \%)$ & $11(24 \%)$ & $2(7 \%)$ & $\mathbf{0 . 0 4 5}{ }^{\mathrm{d}}$ \\
\hline \multicolumn{9}{|c|}{ Distant metastases $(\mathrm{pM}), \mathrm{n}(\%)$} \\
\hline $\mathrm{pM}-$ & $117(91 \%)$ & $69(91 \%)$ & $48(91 \%)$ & & $47(92 \%)$ & $39(87 \%)$ & $27(96 \%)$ & \\
\hline $\mathrm{pM}+$ & $12(9 \%)$ & $7(9 \%)$ & $5(9 \%)$ & $>0.99^{\mathrm{c}}$ & $4(8 \%)$ & $6(13 \%)$ & $1(4 \%)$ & $0.37^{\mathrm{d}}$ \\
\hline \multicolumn{9}{|c|}{ SLNB result $(\mathrm{n}=56), \mathrm{n}(\%)$} \\
\hline No metastases & $35(62 \%)$ & $22(63 \%)$ & $13(62 \%)$ & & $18(35 \%)$ & $10(22 \%)$ & $6(21 \%)$ & \\
\hline Metastases & $21(38 \%)$ & $13(37 \%)$ & $8(38 \%)$ & $>0.99^{\mathrm{c}}$ & $9(18 \%)$ & $8(18 \%)$ & $2(7 \%)$ & $0.63^{\mathrm{d}}$ \\
\hline \multicolumn{9}{|l|}{ Recurrence, n (\%) } \\
\hline No & $85(66 \%)$ & $45(59 \%)$ & $40(75 \%)$ & & $30(59 \%)$ & $30(67 \%)$ & $22(79 \%)$ & \\
\hline Yes & $44(34 \%)$ & $31(41 \%)$ & $13(25 \%)$ & $0.062^{\mathrm{c}}$ & $21(41 \%)$ & $15(33 \%)$ & $6(21 \%)$ & $0.20^{\mathrm{d}}$ \\
\hline
\end{tabular}

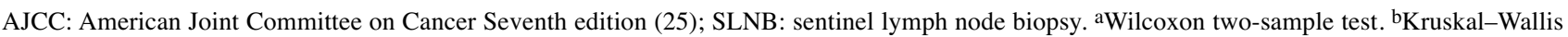
test. 'Fisher's exact test. ${ }^{\mathrm{d}}$ Chi-squared test. Statistically significant results $(p<0.05)$ are shown in bold.

Consistently, the highest ROCK1 and ROCK2 reactivity characterized AJCC stage I tumors (Tables I and II). Comparison of ROCK IRS with microscopic features of skin melanoma revealed more correlations suggestive of downregulation of ROCK1 and ROCK2 expression in advanced and aggressive tumors. Immunoreactivity of both proteins was diminished in thicker, more invasive and mitogenic melanomas. We also noted a predilection for low ROCK1 and ROCK2 levels in a nodular subtype of melanoma, as well as an association with the presence of ulceration. Moreover, ROCK2, but not ROCK1 expression in cancer cells was statistically positively related to the intensity of tumorassociated lymphocytic infiltrate (Tables III and IV).
Expression of ROCK1 and ROCK2 in TILs and clinicopathological characteristics. Strong reactivity for either ROCK1 and ROCK2 in TILs was more frequently found in thinner tumors and cases without concurrent nodal disease. Moreover, weak ROCK2 staining in lymphocytes was associated with melanoma recurrence (Tables I and II). Similarly to the melanocytic expression, the intensity of ROCK staining in TILs was negatively associated with a wide range of microscopic characteristics of primary tumors, e.g. Breslow thickness, Clark level of invasion, mitotic rate and the presence of ulceration. Low ROCK1 and ROCK2 expression was more typical of nodular melanomas. In the case of ROCK1, 70\% of cases with weak staining were 
Table II. Clinical characteristics of melanoma patients according to Rho-associated coiled-coil kinase 2 (ROCK2) expression.

\begin{tabular}{|c|c|c|c|c|c|c|c|c|}
\hline \multirow[t]{3}{*}{ Parameter } & \multirow[b]{3}{*}{$\begin{array}{c}\text { Total } \\
(\mathrm{n}=1299)\end{array}$} & \multicolumn{6}{|c|}{ ROCK2 immunoreactivity } & \\
\hline & & \multicolumn{3}{|c|}{ Melanoma cells } & \multicolumn{4}{|c|}{ Tumor-infiltrating lymphocytes } \\
\hline & & $\begin{array}{l}\text { Low } \\
(\mathrm{n}=60)\end{array}$ & $\begin{array}{l}\text { High } \\
(n=69)\end{array}$ & $p$-Value & $\begin{array}{l}\text { Weak } \\
(\mathrm{n}=28)\end{array}$ & $\begin{array}{c}\text { Medium } \\
(\mathrm{n}=72)\end{array}$ & $\begin{array}{l}\text { Strong } \\
(\mathrm{n}=24)\end{array}$ & $p$-Value \\
\hline \multicolumn{9}{|l|}{ Age, years } \\
\hline Mean \pm SD & $62 \pm 15$ & $60 \pm 14$ & $65 \pm 16$ & $0.024^{\mathrm{a}}$ & $65 \pm 17$ & $61 \pm 15$ & $63 \pm 14$ & $0.57^{\mathrm{b}}$ \\
\hline Median & $65(18-87)$ & $62(18-87)$ & $70(25-85)$ & & $70(18-86)$ & $64.5(24-87)$ & $65.5(33-82)$ & \\
\hline \multicolumn{9}{|l|}{ Gender, n (\%) } \\
\hline Female & $67(52 \%)$ & $27(45 \%)$ & $35(51 \%)$ & & $16(57 \%)$ & $43(60 \%)$ & $6(25 \%)$ & \\
\hline Male & $62(48 \%)$ & $33(55 \%)$ & $34(49 \%)$ & $0.60^{\mathrm{c}}$ & $12(43 \%)$ & $29(40 \%)$ & $18(75 \%)$ & $\mathbf{0 . 0 1 1}{ }^{\mathrm{d}}$ \\
\hline \multicolumn{9}{|c|}{ Primary tumor location, $\mathrm{n}(\%)$} \\
\hline Head/neck & $12(9 \%)$ & $9(15 \%)$ & $3(4 \%)$ & & $6(21 \%)$ & $3(4 \%)$ & $3(12 \%)$ & \\
\hline Extremities & $55(43 \%)$ & $25(42 \%)$ & $30(43 \%)$ & $0.19^{\mathrm{d}}$ & $14(50 \%)$ & $35(49 \%)$ & $4(17 \%)$ & $0.0027^{\mathrm{d}}$ \\
\hline Hand/foot & $4(3 \%)$ & $1(2 \%)$ & $3(4 \%)$ & & $0(0 \%)$ & $3(4 \%)$ & $0(0 \%)$ & \\
\hline Trunk & $58(45 \%)$ & $25(42 \%)$ & $33(48 \%)$ & & $8(29 \%)$ & $31(43 \%)$ & $17(71 \%)$ & \\
\hline \multicolumn{9}{|c|}{ AJCC stage, n (\%) } \\
\hline I & $46(36 \%)$ & $8(13 \%)$ & $38(55 \%)$ & & $4(14 \%)$ & $25(35 \%)$ & $16(67 \%)$ & \\
\hline II & $47(36 \%)$ & $33(55 \%)$ & $14(20 \%)$ & $<0.0001^{\mathrm{d}}$ & $12(43 \%)$ & $28(39 \%)$ & $6(25 \%)$ & $0.0072^{\mathrm{d}}$ \\
\hline III & $24(19 \%)$ & $13(22 \%)$ & $11(16 \%)$ & & $8(29 \%)$ & $12(17 \%)$ & $2(8 \%)$ & \\
\hline IV & $12(9 \%)$ & $6(10 \%)$ & $6(9 \%)$ & & $4(14 \%)$ & $7(10 \%)$ & $0(0 \%)$ & \\
\hline \multicolumn{9}{|c|}{ Primary tumor (pT), n (\%) } \\
\hline pT1 & $30(23 \%)$ & $2(3 \%)$ & $28(41 \%)$ & & $3(11 \%)$ & $14(19 \%)$ & $12(50 \%)$ & \\
\hline pT2 & $22(17 \%)$ & $7(12 \%)$ & $15(22 \%)$ & $<0.0001^{a}$ & $2(7 \%)$ & $14(19 \%)$ & $6(25 \%)$ & $0.0005^{b}$ \\
\hline pT3 & $32(25 \%)$ & $15(25 \%)$ & $17(25 \%)$ & & $7(25 \%)$ & $18(25 \%)$ & $5(21 \%)$ & \\
\hline pT4 & $45(35 \%)$ & $36(60 \%)$ & $9(13 \%)$ & & $16(57 \%)$ & $26(36 \%)$ & $1(4 \%)$ & \\
\hline \multicolumn{9}{|c|}{ Regional lymph node status $(\mathrm{pN}), \mathrm{n}(\%)$} \\
\hline $\mathrm{pN}-$ & $98(76 \%)$ & $43(72 \%)$ & $55(80 \%)$ & & $17(61 \%)$ & $56(78 \%)$ & $22(92 \%)$ & \\
\hline $\mathrm{pN}+$ & $31(24 \%)$ & $17(28 \%)$ & $14(20 \%)$ & $0.31^{\mathrm{c}}$ & $11(39 \%)$ & $16(22 \%)$ & $2(8 \%)$ & $0.034^{\mathrm{d}}$ \\
\hline \multicolumn{9}{|c|}{ Distant metastases $(\mathrm{pM}), \mathrm{n}(\%)$} \\
\hline $\mathrm{pM}-$ & $117(91 \%)$ & $54(90 \%)$ & $63(91 \%)$ & & $24(86 \%)$ & $65(90 \%)$ & $24(100 \%)$ & \\
\hline $\mathrm{pM}+$ & $12(9 \%)$ & $6(10 \%)$ & $6(9 \%)$ & $>0.99^{\mathrm{c}}$ & $4(14 \%)$ & $7(10 \%)$ & $0(0 \%)$ & $0.17^{\mathrm{d}}$ \\
\hline \multicolumn{9}{|c|}{ SLNB result $(\mathrm{n}=56), \mathrm{n}(\%)$} \\
\hline No metastases & $35(62 \%)$ & $18(58 \%)$ & $17(68 \%)$ & & $7(47 \%)$ & $22(69 \%)$ & $5(83 \%)$ & \\
\hline Metastases & $21(38 \%)$ & $13(42 \%)$ & $8(32 \%)$ & $0.58^{\mathrm{c}}$ & $8(53 \%)$ & $10(31 \%)$ & $1(17 \%)$ & $0.21^{\mathrm{d}}$ \\
\hline \multicolumn{9}{|l|}{ Recurrence, n (\%) } \\
\hline No & $85(66 \%)$ & $33(55 \%)$ & $52(75 \%)$ & & $13(46 \%)$ & $48(67 \%)$ & $21(88 \%)$ & \\
\hline Yes & $44(34 \%)$ & $27(45 \%)$ & $17(25 \%)$ & $0.017^{c}$ & $15(54 \%)$ & $24(33 \%)$ & $3(12 \%)$ & $0.0074^{\mathrm{d}}$ \\
\hline
\end{tabular}

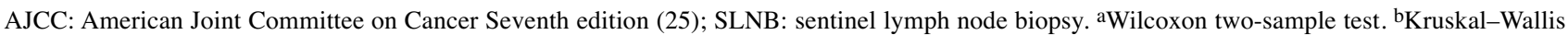
test. 'Fisher's exact test. ${ }^{\mathrm{d}}$ Chi-squared test. Statistically significant results $(p<0.05)$ are shown in bold.

nodular type, while over $70 \%$ of tumors with high expression were superficial spreading melanomas. TIL grade was only weakly associated with ROCK2 immunoreactivity, but no such association was found for ROCK1 (Tables III and IV).

Expression of ROCK1 and ROCK2 and survival of patients with melanoma. To check whether expression of ROCK proteins is associated with clinical outcomes Kaplan-Meier analysis was performed. Although melanocytic ROCK1 immunoreactivity did not stratify the study population with regard to MSS, we noted a trend towards longer RFS in high ROCK1 expressors (Figure 4A). On the other hand, elevated ROCK2 expression in cancer cells characterized patients with favorable MSS and RFS (Figure 4B). Differences in ROCK1 expression in TILs were not associated with patient survival (data not shown) but stronger ROCK2 staining was significantly related to longer MSS and RFS (Figure 4C). However, neither tumoral nor lymphocytic expression of ROCK2 were independent predictors of survival when adjusted for Breslow thickness and $\mathrm{pN}$ status in multivariable regression models (data not shown).

\section{Discussion}

The RhoA-ROCK pathway is a master regulator of cytoskeletal dynamics, influencing multiple cell functions, 
Table III. Microscopic features of primary melanoma tumors and Rho-associated coiled-coil kinase 1 (ROCK1) expression.

\begin{tabular}{|c|c|c|c|c|c|c|c|c|}
\hline \multirow[t]{3}{*}{ Parameter } & \multirow[b]{3}{*}{$\begin{array}{c}\text { Total } \\
(n=129)\end{array}$} & \multicolumn{7}{|c|}{ ROCK1 immunoreactivity } \\
\hline & & \multicolumn{3}{|c|}{ Melanoma cells } & \multicolumn{4}{|c|}{ Tumor-infiltrating lymphocytes } \\
\hline & & $\begin{array}{l}\text { Low } \\
(\mathrm{n}=76)\end{array}$ & $\begin{array}{c}\text { High } \\
(\mathrm{n}=536)\end{array}$ & $p$-Value & $\begin{array}{c}\text { Weak } \\
(\mathrm{n}=516)\end{array}$ & $\begin{array}{l}\text { Medium } \\
(\mathrm{n}=456)\end{array}$ & $\begin{array}{c}\text { Strong } \\
(\mathrm{n}=286)\end{array}$ & $p$-Value \\
\hline \multicolumn{9}{|l|}{ Breslow thickness, mm } \\
\hline Mean \pm SD & $5.1 \pm 6.3$ & $6.3 \pm 6.2$ & $3.3 \pm 6.3$ & $<0.0001^{\mathrm{a}}$ & $7.2 \pm 7.0$ & $4.3 \pm 6.4$ & $2.5 \pm 3.3$ & $0.0014^{b}$ \\
\hline Median & $2.7(0.3-40)$ & $3.7(0.4-30)$ & $1.5(0.3-40)$ & & $4.7(0.4-30)$ & $2.3(0.5-40)$ & $1.2(0.3-15)$ & \\
\hline \multicolumn{9}{|l|}{ Clark level, n (\%) } \\
\hline II & $37(29 \%)$ & $11(14 \%)$ & $26(49 \%)$ & & $8(16 \%)$ & $14(31 \%)$ & $14(50 \%)$ & \\
\hline III & $41(32 \%)$ & $24(32 \%)$ & $17(32 \%)$ & & $19(37 \%)$ & $13(29 \%)$ & $9(32 \%)$ & \\
\hline IV & $36(28 \%)$ & $31(41 \%)$ & $5(9 \%)$ & & $18(35 \%)$ & $10(22 \%)$ & $5(18 \%)$ & \\
\hline $\mathrm{V}$ & $15(12 \%)$ & $10(13 \%)$ & $5(9 \%)$ & $<0.0001^{\mathrm{a}}$ & $6(12 \%)$ & $8(18 \%)$ & $0(0 \%)$ & $0.014^{b}$ \\
\hline \multicolumn{9}{|l|}{ Histological type, n (\%) } \\
\hline Superficial spreading & $58(45 \%)$ & $20(26 \%)$ & $38(72 \%)$ & & $14(27 \%)$ & $22(49 \%)$ & $20(71 \%)$ & \\
\hline Nodular & $67(52 \%)$ & $53(70 \%)$ & $14(26 \%)$ & & $36(71 \%)$ & $21(47 \%)$ & $8(29 \%)$ & \\
\hline Acral-lentiginous & $4(3 \%)$ & $3(4 \%)$ & $1(2 \%)$ & $<0.0001^{\mathrm{c}}$ & $1(2 \%)$ & $2(4 \%)$ & $0(0 \%)$ & $0.0012^{c}$ \\
\hline \multicolumn{9}{|l|}{ Mitotic rate, n (\%) } \\
\hline 0 & $33(26 \%)$ & $9(12 \%)$ & $24(45 \%)$ & & $9(18 \%)$ & $11(24 \%)$ & $12(43 \%)$ & \\
\hline $1-2$ & $20(16 \%)$ & $9(12 \%)$ & $11(21 \%)$ & & $3(6 \%)$ & $9(20 \%)$ & $6(21 \%)$ & \\
\hline$>2$ & $76(59 \%)$ & $58(76 \%)$ & $18(34 \%)$ & $<0.0001^{\mathrm{c}}$ & $39(76 \%)$ & $25(56 \%)$ & $10(36 \%)$ & $0.0051^{c}$ \\
\hline \multicolumn{9}{|l|}{ Ulceration, n (\%) } \\
\hline No & $76(59 \%)$ & $37(49 \%)$ & $39(74 \%)$ & & $21(41 \%)$ & $32(71 \%)$ & $19(68 \%)$ & \\
\hline Yes & $53(41 \%)$ & $39(51 \%)$ & $14(26 \%)$ & $0.0062^{\mathrm{d}}$ & $30(59 \%)$ & $13(29 \%)$ & $9(32 \%)$ & $0.0062^{c}$ \\
\hline \multicolumn{9}{|l|}{ TILs, n (\%) } \\
\hline Absent & $5(4 \%)$ & $4(5 \%)$ & $1(2 \%)$ & & $\mathrm{n} / \mathrm{a}$ & $\mathrm{n} / \mathrm{a}$ & $\mathrm{n} / \mathrm{a}$ & \\
\hline Non-brisk & $81(63 \%)$ & $51(67 \%)$ & $30(57 \%)$ & & $38(75 \%)$ & $29(64 \%)$ & $14(50 \%)$ & \\
\hline Brisk & $43(33 \%)$ & $21(28 \%)$ & $22(42 \%)$ & $0.25^{\mathrm{c}}$ & $13(25 \%)$ & $16(36 \%)$ & $14(50 \%)$ & $0.094^{\mathrm{c}}$ \\
\hline \multicolumn{9}{|l|}{ Microsatellites, n (\%) } \\
\hline No & $123(95 \%)$ & $71(93 \%)$ & $52(98 \%)$ & & $48(94 \%)$ & $42(93 \%)$ & $28(100 \%)$ & \\
\hline Yes & $6(5 \%)$ & $5(7 \%)$ & $1(2 \%)$ & $0.40^{\mathrm{d}}$ & $3(6 \%)$ & $3(7 \%)$ & $0(0 \%)$ & $0.50^{\mathrm{c}}$ \\
\hline \multicolumn{9}{|c|}{ Lymphovascular invasion, $\mathrm{n}(\%)$} \\
\hline No & $121(94 \%)$ & $71(93 \%)$ & $50(94 \%)$ & & $46(90 \%)$ & $43(96 \%)$ & $28(100 \%)$ & \\
\hline Yes & $8(6 \%)$ & $5(7 \%)$ & $3(6 \%)$ & $>0.99 \mathrm{~d}$ & $5(10 \%)$ & $2(4 \%)$ & $0(0 \%)$ & $0.18^{\mathrm{c}}$ \\
\hline
\end{tabular}

TILs: Tumor-infiltrating lymphocytes. ${ }^{a}$ Wilcoxon two-sample test. ${ }^{b}$ Kruskal-Wallis test. ${ }^{c}$ Chi-squared test. ${ }^{d}$ Fisher's exact test. Statistically significant results $(p<0.05)$ are shown in bold.

such as polarity, movement and proliferation (1). Consequently, many studies argued for an oncogenic role of Rho-ROCK signaling in various cancer types, especially during invasion and metastasis (2). In our previous study, we demonstrated on clinical samples that RhoA is downregulated rather than overexpressed in advanced and aggressive skin melanomas (28). Here, we tested whether expression of effector kinases intimately regulated by RhoA, i.e. ROCK1 and ROCK2, is also related to the tumor stage and other clinicopathological features. We found a striking predilection for low melanocytic immunoreactivity of both kinases in thick, deeply invasive, ulcerated and mitogenic tumors. Moreover, ROCK1 and -2 were preferentially downregulated in nodular melanomas and diminished ROCK2 expression was associated with ominous prognosis. This apparent suppression of the RhoA-ROCK pathway that occurs in aggressive and late-stage tumors suggests its inhibitory role in melanomagenesis, which is contrary to the majority of reports concerning other cancer types.

The current notion of the role that ROCKs play in skin melanoma originate from a number of mechanistic experiments involving cell cultures and murine models. However, the particularities of each study, i.e. focus on different specialized contexts, utilization of different ROCKblocking agents and, notably, different cell lines, resulted in discordant conclusions. Most investigations used B16 mouse melanoma cells and showed that ROCK inhibition impaired tumor growth, invasiveness and formation of metastases (3, 29-32). While observations of human A375 cells and several uveal melanoma lines corroborated these results $(3,30)$, ROCK inhibition in HT-144 cells was associated with a proinvasive effect (33). This lability was recently evidenced by 
Table IV. Microscopic features of primary melanoma tumors and Rho-associated coiled-coil kinase 2 (ROCK2) expression.

\begin{tabular}{|c|c|c|c|c|c|c|c|c|}
\hline \multirow[t]{3}{*}{ Parameter } & \multirow[b]{3}{*}{$\begin{array}{c}\text { Total } \\
(\mathrm{n}=129)\end{array}$} & \multicolumn{7}{|c|}{ ROCK2 immunoreactivity } \\
\hline & & \multicolumn{3}{|c|}{ Melanoma cells } & \multicolumn{4}{|c|}{ Tumor-infiltrating lymphocytes } \\
\hline & & $\begin{array}{l}\text { Low } \\
(\mathrm{n}=60)\end{array}$ & $\begin{array}{c}\text { High } \\
(\mathrm{n}=69)\end{array}$ & $p$-Value & $\begin{array}{c}\text { Weak } \\
(\mathrm{n}=28)\end{array}$ & $\begin{array}{c}\text { Medium } \\
(\mathrm{n}=72)\end{array}$ & $\begin{array}{l}\text { Strong } \\
(\mathrm{n}=24)\end{array}$ & $p$-Value \\
\hline \multicolumn{9}{|l|}{ Breslow thickness, mm } \\
\hline Mean \pm SD & $5.1 \pm 6.3$ & $7.8 \pm 6.6$ & $2.7 \pm 5.0$ & $<0.0001^{\mathrm{a}}$ & $7.8 \pm 7.0$ & $5.1 \pm 6.6$ & $1.7 \pm 2.0$ & $0.0005^{b}$ \\
\hline Median & $2.7(0.3-40)$ & $4.9(0.6-30)$ & $1.5(0.3-40)$ & & $4.4(0.6-23)$ & $2.8(0.3-40)$ & $1.0(0.3-10)$ & \\
\hline \multicolumn{9}{|l|}{ Clark level, n (\%) } \\
\hline II & $37(29 \%)$ & $3(5 \%)$ & $34(49 \%)$ & & $3(11 \%)$ & $18(25 \%)$ & $15(63 \%)$ & \\
\hline III & $41(32 \%)$ & $20(33 \%)$ & $21(30 \%)$ & & $8(29 \%)$ & $26(36 \%)$ & $7(29 \%)$ & \\
\hline IV & $36(28 \%)$ & $25(42 \%)$ & $11(16 \%)$ & & $9(32 \%)$ & $22(31 \%)$ & $2(8 \%)$ & \\
\hline $\mathrm{V}$ & $15(12 \%)$ & $12(20 \%)$ & $3(4 \%)$ & $<0.0001^{a}$ & $8(29 \%)$ & $6(8 \%)$ & $0(0 \%)$ & $0.0002^{b}$ \\
\hline \multicolumn{9}{|l|}{ Histological type, n (\%) } \\
\hline Superficial spreading & $58(45 \%)$ & $9(15 \%)$ & $49(71 \%)$ & & $5(18 \%)$ & $30(42 \%)$ & $21(88 \%)$ & \\
\hline Nodular & $67(52 \%)$ & $50(83 \%)$ & $17(25 \%)$ & & $23(82 \%)$ & $39(54 \%)$ & $3(12 \%)$ & \\
\hline Acral-lentiginous & $4(3 \%)$ & $1(2 \%)$ & $3(4 \%)$ & $<0.0001^{\mathrm{c}}$ & $0(0 \%)$ & $3(4 \%)$ & $0(0 \%)$ & $<0.0001^{\mathrm{c}}$ \\
\hline \multicolumn{9}{|l|}{ Mitotic rate, n (\%) } \\
\hline 0 & $33(26 \%)$ & $2(3 \%)$ & $31(45 \%)$ & & $4(14 \%)$ & $15(21 \%)$ & $13(54 \%)$ & \\
\hline $1-2$ & $20(16 \%)$ & $6(10 \%)$ & $14(20 \%)$ & & $1(4 \%)$ & $11(15 \%)$ & $6(25 \%)$ & \\
\hline$>2$ & $76(59 \%)$ & $52(87 \%)$ & $24(35 \%)$ & $<0.0001^{\mathrm{c}}$ & $23(82 \%)$ & $46(64 \%)$ & $5(21 \%)$ & $0.0001^{c}$ \\
\hline \multicolumn{9}{|l|}{ Ulceration, n (\%) } \\
\hline No & $76(59 \%)$ & $22(37 \%)$ & $54(78 \%)$ & & $12(43 \%)$ & $41(57 \%)$ & $19(79 \%)$ & \\
\hline Yes & $53(41 \%)$ & $38(63 \%)$ & $15(22 \%)$ & $<0.0001^{\mathrm{d}}$ & $16(57 \%)$ & $31(43 \%)$ & $5(21 \%)$ & $0.030^{c}$ \\
\hline \multicolumn{9}{|l|}{ TILs, n (\%) } \\
\hline Absent & $5(4 \%)$ & $1(2 \%)$ & $4(6 \%)$ & & $\mathrm{n} / \mathrm{a}$ & $\mathrm{n} / \mathrm{a}$ & $\mathrm{n} / \mathrm{a}$ & \\
\hline Non-brisk & $81(63 \%)$ & $48(80 \%)$ & $33(48 \%)$ & & $22(79 \%)$ & $48(67 \%)$ & $11(46 \%)$ & \\
\hline Brisk & $43(33 \%)$ & $11(18 \%)$ & $32(46 \%)$ & $0.0004^{c}$ & $6(21 \%)$ & $24(33 \%)$ & $13(54 \%)$ & $0.048^{\mathrm{c}}$ \\
\hline \multicolumn{9}{|l|}{ Microsatellitosis, n (\%) } \\
\hline No & $123(95 \%)$ & $56(93 \%)$ & $67(97 \%)$ & & $25(89 \%)$ & $69(96 \%)$ & $24(100 \%)$ & \\
\hline Yes & $6(5 \%)$ & $4(7 \%)$ & $2(3 \%)$ & $0.42^{\mathrm{d}}$ & $3(11 \%)$ & $3(4 \%)$ & $0(0 \%)$ & $0.20^{\mathrm{c}}$ \\
\hline \multicolumn{9}{|c|}{ Lymphovascular invasion, $\mathrm{n}(\%)$} \\
\hline No & $121(94 \%)$ & $55(92 \%)$ & $66(96 \%)$ & & $23(82 \%)$ & $70(97 \%)$ & $24(100 \%)$ & \\
\hline Yes & $8(6 \%)$ & $5(8 \%)$ & $3(4 \%)$ & $0.47^{\mathrm{d}}$ & $5(18 \%)$ & $2(3 \%)$ & $0(0 \%)$ & $0.010^{\mathrm{c}}$ \\
\hline
\end{tabular}

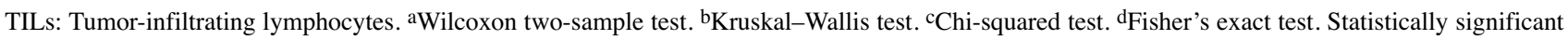
results $(p<0.05)$ are shown in bold.

Chang et al. who compared consequences of ROCK inhibition across several cell lines (22). Treatment with Y27632 reduced proliferation and migration in $\mathrm{B} 16 \mathrm{~F} 1$ and MeWo cells, but employment of UACC257, UACC62 and M14 lines yielded contrary results, which our findings are consistent with (22). This variation was attributed to B-Raf proto-oncogene, serine/threonine kinase (BRAF) status as only $B R A F$-mutant cells displayed enhanced growth and motility following ROCK inhibition (22). Since BRAF mutations are found in around $60 \%$ of skin melanomas (34), the peculiarity of ROCK function in these cases may be a major factor influencing the role of ROCK 1 or ROCK2 in the clinical conditions. Interestingly, other groups revealed resistance to BRAF inhibitors to be associated with upregulation of RhoA/ROCK signaling and that coordinated inhibition of BRAF and ROCK may be therapeutically appealing $(35,36)$. Overall, the significance of ROCK signaling appears to be highly cell- and context-dependent, and individual experimental models likely do not mirror the complexity and heterogeneity of clinical disease. To the best of our knowledge, our results are the first documentation of clinicopathological profiles of ROCK1 and -2 protein expression in patient-derived melanoma samples.

The RhoA-ROCK pathway is known to affect immune responses (24); therefore, we decided to include TILs in the evaluation of ROCK1 and -2 expression in primary melanomas. Since RhoA-ROCK signaling is required for migration of lymphocytes through the endothelial barrier and perivascular collagen $(23,37)$, we anticipated a relationship between TIL grade and ROCK reactivity. However, only ROCK2 expression in TILs was positively correlated with the intensity of lymphocytic infiltrate, and yet even this association was weak. Nevertheless, we observed significant correlations between lymphocytic staining for either ROCK1 


\section{Melanoma-specific survival}

\section{Recurrence-free survival}

\section{A ROCK1}
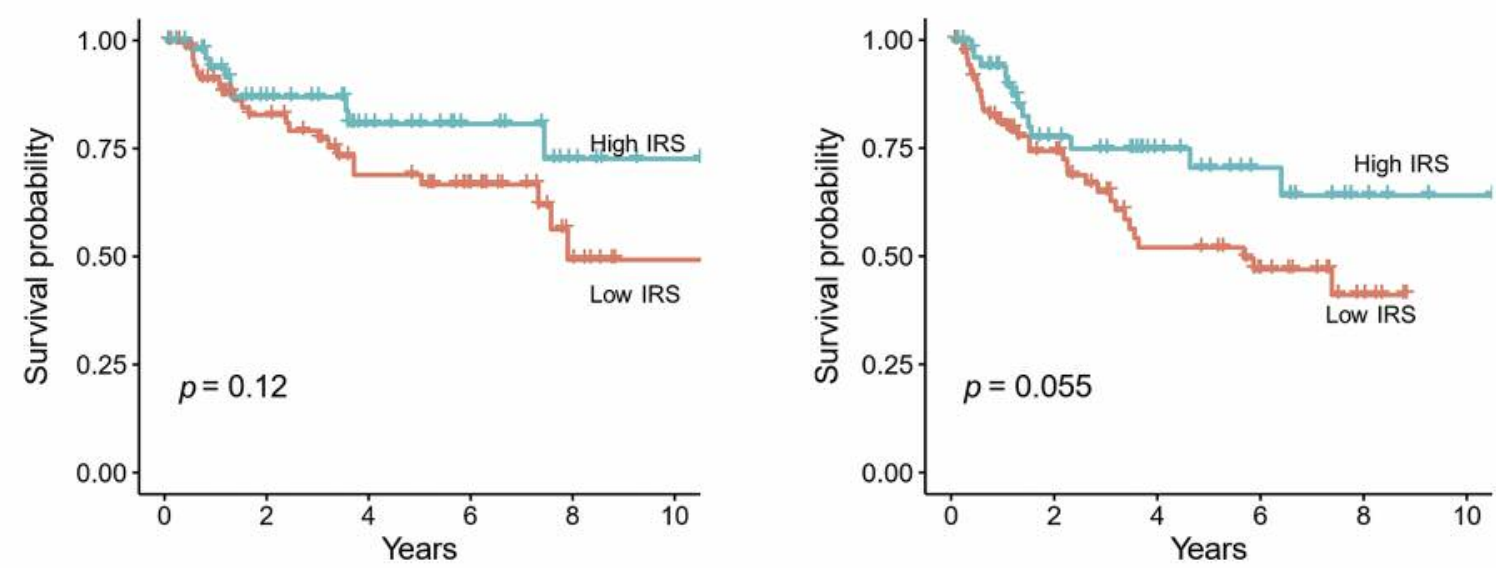

\section{B ROCK2}
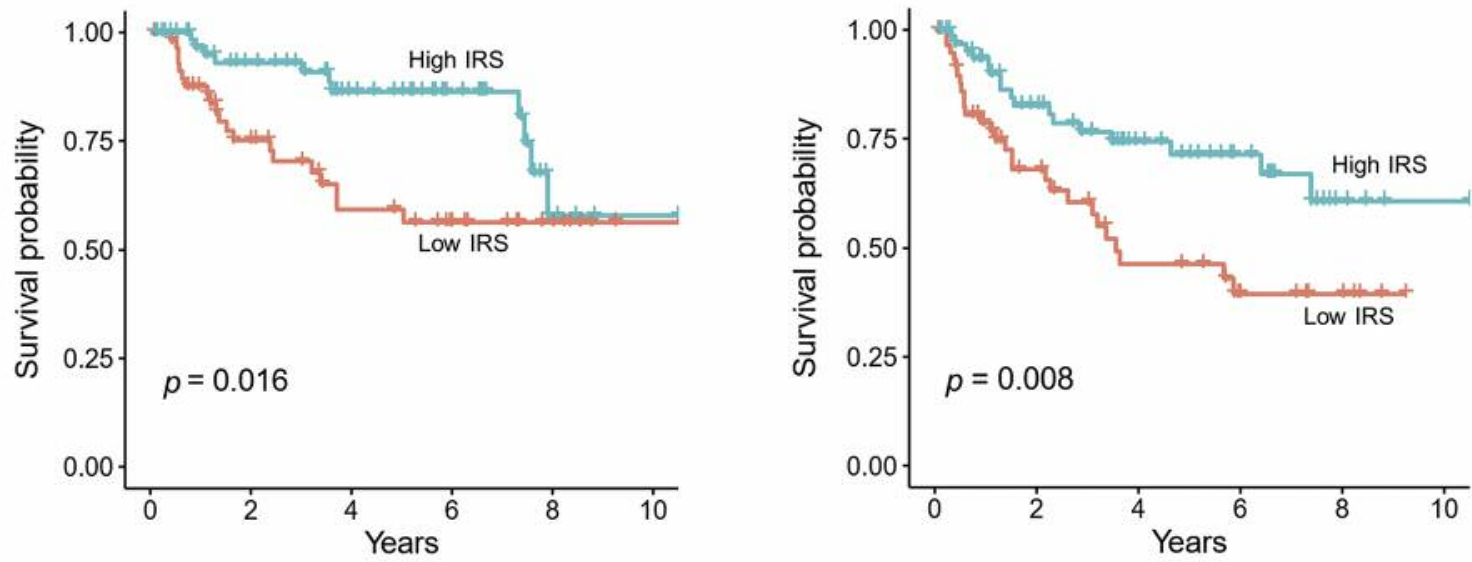

\section{TILs ROCK2}
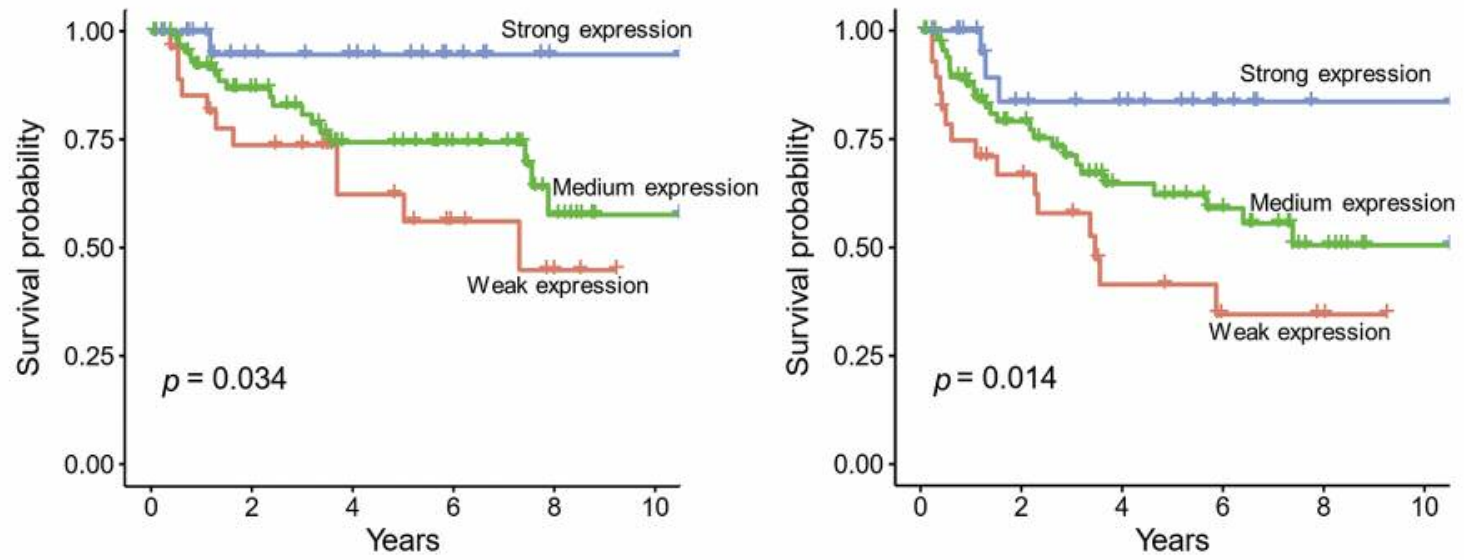

Figure 4. Kaplan-Meier plots representing patient survival according to characteristics of Rho-associated coiled-coil kinase (ROCK) 1 and 2 expression in primary melanoma tumors. Differences between melanocytic expression of ROCK1 were not related to clinical outcome (A), but lower melanocytic expression of ROCK2 characterized patients with significantly shorter melanoma-specific survival and recurrence-specific survival (B, respectively). Moreover, reactivity for ROCK2 in tumor-infiltrating lymphocytes (TILs) positively correlated with patient prognosis (C). 
and -2 and other characteristics of melanoma, e.g. Breslow thickness, histological type, mitotic rate or ulceration. Overall, both proteins were expressed more strongly in tumors with favorable histological features and low clinical advancement. Interestingly, cases with nodal metastases at presentation accounted for $7 \%$ and $8 \%$ of tumors with strong lymphocytic ROCK 1 and ROCK 2 expression, respectively, but these rates escalated to $31 \%$ and $39 \%$ for melanomas with weak staining for ROCK1 and ROCK2 in TILs, respectively. In addition, reduced ROCK 2 expression in TILs correlated with poor prognosis. What seems an alluring hypothesis is that TILs with higher activity of ROCKs are more effective at disease containment. This would be in line with previous studies reporting involvement of the RhoROCK pathway in T-cell activation and cytotoxicity (38-40). The role of ROCKs expressed by tumor-reactive immune cells remain virtually unexplored but our study indicates that it may be involved in the pathogenesis of cancer, and thus merits further investigation.

ROCK1 and ROCK2 are highly homologous isomers with serine/threonine kinase activity, sharing $65 \%$ of their entire amino acid sequence and over $90 \%$ of the catalytic domain (1). Both proteins have traditionally been considered to play similar roles in the control of cytoskeletal dynamics, cellcycle progression and cellular adhesion (1). More recently, development of selective inhibitors and techniques of isoform-specific ROCK depletion allowed for appreciation of nonsynonymous functions of ROCK 1 and -2 in various settings, including cancer. In one study, inhibition of ROCK2, but not ROCK1, triggered invasion of clustered colorectal carcinoma cells, and reduced ROCK2 mRNA correlated with shorter patient survival, whereas no such correlation was observed for ROCK1 expression (13). Functional investigations of glioblastoma cells revealed that selective knockdown of ROCK1 or ROCK2 exerted opposite effects on proliferation (41). Moreover, inhibition of ROCK1 or ROCK2 reduced or enhanced, respectively, glioblastoma cell migration on laminin-coated surface (41). Wang et al. demonstrated mRNA and protein expression of ROCK1 and ROCK2 in Y79 retinoblastoma cells but inhibition of only the former isoform affected cell adhesion and invasion (42). Most of the limited studies related to ROCK signaling in melanoma did not aim to differentiate between the roles of ROCK1 and -2 isoforms but Kümper et al. compared them in a mouse model of $B R A F$-mutated melanoma. Conditional knockout of Rock 2 resulted in earlier onset of tumorigenesis and reduced survival when compared with depletion of Rock1 or both genes (43). In our study, expression of either ROCK1 and -2 was negatively correlated with Breslow thickness, ulceration and mitotic index. High analogy between ROCK1 and ROCK2 with regard to these clinicopathological associations advocate for a rather similar role of these proteins in the clinical setting. Moreover, melanocytic immunoreactivity of ROCK1 was moderately correlated to ROCK2 throughout the entire cohort with a coefficient of 0.63 between IRS for ROCK1 and ROCK2. Thus, mechanisms that regulate cellular concentrations of either ROCK isoform may be at least partially mutual. Contrarily, neither ROCK1 nor ROCK2 expression correlated with RhoA reactivity in our cohort. Such associations were reported previously in testicular and bladder tumors $(44,45)$, as well as in breast cancer, in which coordinated expression of RhoA and ROCKs was induced by hypoxia (46). Our results suggest that in melanoma, transcription or cellular turnover of RhoA and ROCK isoforms are regulated independently.

In summary, this and our previous studies demonstrated that constituents of Rho-ROCK pathway are down-regulated in aggressive and clinically advanced melanomas (28). Although definitive conclusions about the mechanistic involvement of ROCK 1 and -2 in melanoma pathogenesis cannot be directly drawn from analysis of protein expression, our results raise the question of whether prior observations based on experiments with cell lines may be easily translatable into clinically valid conclusions. Comparison of ROCK1 and -2 expression in primary and metastatic melanoma lesions, as well as clinical verification of the relationship between BRAF status and RhoA-ROCK signaling, may help clarify the potential for therapeutic modulation of this pathway in cutaneous melanoma.

\section{Conflicts of Interest}

The Authors report no conflicts of interest in regard to this study.

\section{Authors' Contributions}

MK: Study design, resources, evaluation of immunohistochemistry, analysis of the results, drafting the article; PB: resources, statistical analysis, analysis of the results; MP: clinical data, analysis of the results; PD: resources, evaluation of immunohistochemistry, analysis of the results; RM: resources, analysis of the results; $\mathrm{AH}$ : analysis of the results, supervision. All Authors read, revised and approved the final article.

\section{Acknowledgements}

This study was funded by a statutory subsidy by the Polish Ministry of Science and Higher Education as part of grants STM.B131.17.008, SUB.C280.19.050 and ST.B130.18.030 (record numbers in the Simple system); PB was financially supported by the National Science Centre grant Opus 2016/21/B/ST6/02176.

\section{References}

1 Julian L and Olson MF: Rho-associated coiled-coil containing kinases (ROCK). Small GTPases 5(2): e29846, 2014. PMID: 25010901. DOI: $10.4161 /$ sgtp.29846 
2 Wei L, Surma M, Shi S, Lambert-Cheatham N and Shi J: Novel Insights into the roles of Rho kinase in cancer. Arch Immunol Ther Exp 64(4): 259-278, 2016. PMID: 26725045. DOI: 10.1007/s00005-015-0382-6

3 Huang G, Wang Y, Su J, Zhou P, Li B, Yin L and Lu J: Upregulation of Rho-associated kinase $1 / 2$ by glucocorticoids promotes migration, invasion and metastasis of melanoma. Cancer Lett 410: 1-11, 2017. PMID: 28923399. DOI: 10.1016/j.canlet.2017.09.005

4 Sadok A, McCarthy A, Caldwell J, Collins I, Garrett MD, Yeo M, Hooper S, Sahai E, Kuemper S, Mardakheh FK and Marshall CJ: Rho kinase inhibitors block melanoma cell migration and inhibit metastasis. Cancer Res 75(11): 2272-2284, 2015. PMID: 25840982. DOI: $10.1158 / 0008-5472$.CAN-14-2156

5 Liu S, Goldstein RH, Scepansky EM and Rosenblatt M: Inhibition of Rho-associated kinase signaling prevents breast cancer metastasis to human bone. Cancer Res 69(22): 8742-8751, 2009. PMID: 19887617. DOI: 10.1158/0008-5472.CAN-09-1541

6 Whatcott CJ, Ng S, Barrett MT, Hostetter G, Von Hoff DD and Han H: Inhibition of ROCK1 kinase modulates both tumor cells and stromal fibroblasts in pancreatic cancer. PLoS One 12(8). e0183871, 2017. PMID: 28841710. DOI: 10.1371/journal. pone. 0183871

7 Mikuriya Y, Tashiro H, Kuroda S, Nambu J, Kobayashi T, Amano H, Tanaka $\mathrm{Y}$ and Ohdan $\mathrm{H}$ : Fatty liver creates a prometastatic microenvironment for hepatocellular carcinoma through activation of hepatic stellate cells. Int J Cancer 136(4): E3-E13, 2015. PMID: 25053237. DOI: 10.1002/ijc.29096

8 Jeong KJ, Park SY, Cho KH, Sohn JS, Lee J, Kim YK, Kang J, Park CG, Han JW and Lee HY: The Rho/ROCK pathway for lysophosphatidic acid-induced proteolytic enzyme expression and ovarian cancer cell invasion. Oncogene 31(39): 4279-4289, 2012. PMID: 22249252. DOI: 10.1038/onc.2011.595

9 Wermke M, Camgoz A, Paszkowski-Rogacz M, Thieme S, von Bonin M, Dahl A, Platzbecker U, Theis M, Ehninger G, Brenner $\mathrm{S}$, Bornhäuser $\mathrm{M}$ and Buchholz $\mathrm{F}$ : RNAi profiling of primary human AML cells identifies ROCK 1 as a therapeutic target and nominates fasudil as an antileukemic drug. Blood 125(24). 3760-3768, 2015. PMID: 25931586. DOI: 10.1182/blood-201407-590646

10 Abe H, Kamai T, Hayashi K, Anzai N, Shirataki H, Mizuno T, Yamaguchi Y, Masuda A, Yuki H, Betsunoh H, Yashi M, Fukabori Y and Yoshida K-I: The Rho-kinase inhibitor HA-1077 suppresses proliferation/migration and induces apoptosis of urothelial cancer cells. BMC Cancer 14(1): 412, 2014. PMID: 24908363. DOI: $10.1186 / 1471-2407-14-412$

11 Routhier A, Astuccio M, Lahey D, Monfredo N, Johnson A, Callahan W, Partington A, Fellows K, Ouellette L, Zhidro S Goodrow C, Smith A, Sullivan K, Simone P, Le L, Vezuli B, Zohni M, West E, Gleason D and Bryan B: Pharmacological inhibition of Rho-kinase signaling with Y-27632 blocks melanoma tumor growth. Oncol Rep 23(3): 861-867, 2010. PMID: 21109980. DOI: $10.3892 /$ or-00000708

12 Yang S and Kim H-M: ROCK inhibition activates MCF-7 cells. PLoS One 9(2): e88489, 2014. PMID: 24523903. DOI: 10.1371/journal.pone.0088489

13 Libanje F, Raingeaud J, Luan R, Thomas Z, Zajac O, Veiga J, Marisa L, Adam J, Boige V, Malka D, Goéré D, Hall A, Soazec J, Prall F, Gelli M, Dartigues P and Jaulin F: ROCK 2 inhibition triggers the collective invasion of colorectal adenocarcinomas.
EMBO J 38(14): e99299, 2019. PMID: 31304629. DOI: 10.15252/embj.201899299

14 Nakashima M, Adachi S, Yasuda I, Yamauchi T, Kozawa O and Moriwaki H: Rho-kinase regulates negatively the epidermal growth factor-stimulated colon cancer cell proliferation. Int $\mathrm{J}$ Oncol 36(3): 585-592, 2010. PMID: 20126978. DOI: 10.3892/ijo_00000533

15 Nakashima M, Adachi S, Yasuda I, Yamauchi T, Kawaguchi J, Hanamatsu T, Yoshioka T, Okano Y, Hirose Y, Kozawa O and Moriwaki H: Inhibition of Rho-associated coiled-coil containing protein kinase enhances the activation of epidermal growth factor receptor in pancreatic cancer cells. Mol Cancer 10(1): 79, 2011. PMID: 21722395. DOI: 10.1186/1476-4598-10-79

16 Street CA, Routhier AA, Spencer C, Perkins AL, Masterjohn K, Hackathorn A, Montalvo J, Dennstedt EA and Bryan BA: Pharmacological inhibition of Rho-kinase (ROCK) signaling enhances cisplatin resistance in neuroblastoma cells. Int J Oncol 37(5): 1297-1305, 2010. PMID: 20878077. DOI: 10.3892/ijo_ 00000781

17 Li G, Liu L, Shan C, Cheng Q, Budhraja A, Zhou T, Cui H and Gao N: RhoA/ROCK/PTEN signaling is involved in AT-101mediated apoptosis in human leukemia cells in vitro and in vivo. Cell Death Dis 5(1): e998-e998, 2014. PMID: 24434521. DOI: 10.1038/cddis.2013.519

18 Yin $\mathrm{Z}$ and Sun J: Curcumin induces human SKOV3 cell apoptosis via the activation of Rho-kinase. Eur J Gynaecol Oncol 35(4): 433-437, 2014. PMID: 25118487. DOI: 10.12892/ejgo24912014

19 Ohata H, Ishiguro T, Aihara Y, Sato A, Sakai H, Sekine S, Taniguchi H, Akasu T, Fujita S, Nakagama $\mathrm{H}$ and Okamoto K: induction of the stem-like cell regulator CD44 by Rho kinase inhibition contributes to the maintenance of colon cancerinitiating cells. Cancer Res 72(19): 5101-5110, 2012. PMID: 23002207. DOI: 10.1158/0008-5472.CAN-11-3812

20 Tilson SG, Haley EM, Triantafillu UL, Dozier DA, Langford CP, Gillespie GY and Kim Y: ROCK Inhibition facilitates in vitro expansion of glioblastoma stem-like cells. PLoS One 10(7): e0132823, 2015. PMID: 26167936. DOI: 10.1371/journal.pone. 0132823

21 Xia Y, Cai X-Y, Fan J-Q, Zhang L-L, Ren J-H, Chen J, Li Z-Y, Zhang R-G, Zhu F and Wu G: Rho kinase inhibitor fasudil suppresses the vasculogenic mimicry of B16 mouse melanoma cells both in vitro and in vivo. Mol Cancer Ther 14(7): 1582-1590, 2015. PMID: 25934709. DOI: 10.1158/1535-7163.MCT-14-0523

22 Chang F, Zhang Y, Mi J, Zhou Q, Bai F, Xu X, Fisher DE, Sun Q and $\mathrm{Wu} X$ : ROCK inhibitor enhances the growth and migration of BRAF-mutant skin melanoma cells. Cancer Sci 109(11): 34283437, 2018. PMID: 30168234. DOI: 10.1111/cas.13786

23 Ricker E, Chowdhury L, Yi W and Pernis AB: The RhoA-ROCK pathway in the regulation of $\mathrm{T}$ - and $\mathrm{B}$-cell responses. F1000Research 5: 2295, 2016. PMID: 27785353. DOI: 10.12688/f1000research.7522.1

24 Bros M, Haas K, Moll L and Grabbe S: RhoA as a key regulator of innate and adaptive immunity. Cells 8(7): 733, 2019. PMID: 31319592. DOI: 10.3390/cells8070733

25 Balch CM, Gershenwald JE, Soong SJ, Thompson JF, Atkins MB, Byrd DR, Buzaid AC, Cochran AJ, Coit DG, Ding S, Eggermont AM, Flaherty KT, Gimotty PA, Kirkwood JM, McMasters KM, Mihm MC, Morton DL, Ross MI, Sober AJ and Sondak VK: Final version of 2009 AJCC melanoma staging and 
classification. J Clin Oncol 27(36): 6199-6206, 2009. PMID: 19917835. DOI: $10.1200 / J C O .2009 .23 .4799$

26 Remmele W and Stegner HE: Recommendation for uniform definition of an immunoreactive score (IRS) for immunohistochemical estrogen receptor detection (ER-ICA) in breast cancer tissue. Pathologe 8(3): 138-140, 1987. PMID: 3303008.

27 Kaczorowski M, Biecek P, Donizy P, Pieniazek M, Matkowski $\mathrm{R}$ and Halon A: SMAD7 is a novel independent predictor of survival in patients with cutaneous melanoma. Transl Res 204: 72-81, 2018. PMID: 30342000. DOI: 10.1016/j.trsl.2018.09.002

28 Kaczorowski M, Biecek P, Donizy P, Pieniazek M, Matkowski $\mathrm{R}$ and Halon A: Low RhoA expression is associated with adverse outcome in melanoma patients: A clinicopathological analysis. Am J Transl Res 11(7): 4524-4532, 2019. PMID: 31396356.

29 Teiti I, Florie B, Pich C, Gence R, Lajoie-Mazenc I, Rochaix P, Favre $\mathrm{G}$ and Tilkin-Mariamé A-F: In vivo effects in melanoma of ROCK inhibition-induced FasL overexpression. Front Oncol 5: 156, 2015. PMID: 26236689. DOI: 10.3389/fonc.2015.00156

30 Routhier A, Astuccio M, Lahey D, Monfredo N, Johnson A, Callahan W, Partington A, Fellows K, Ouellette L, Zhidro S, Goodrow C, Smith A, Sullivan K, Simone P, Le L, Vezuli B, Zohni M, West E, Gleason D and Bryan B: Pharmacological inhibition of Rho-kinase signaling with Y-27632 blocks melanoma tumor growth. Oncol Rep 23(3): 861-867, 2010. PMID: 20127030. DOI: 10.3892/or_00000708

31 Kidera Y, Tsubaki M, Yamazoe Y, Shoji K, Nakamura H, Ogaki M, Satou T, Itoh T, Isozaki M, Kaneko J, Tanimori Y, Yanae M and Nishida S: Reduction of lung metastasis, cell invasion, and adhesion in mouse melanoma by statin-induced blockade of the Rho/Rho-associated coiled-coil-containing protein kinase pathway. J Exp Clin Cancer Res 29(1): 127, 2010. PMID: 20843370. DOI: $10.1186 / 1756-9966-29-127$

32 Nakajima M, Hayashi K, Egi Y, Katayama K, Amano Y, Uehata M, Ohtsuki M, Fujii A, Oshita K, Kataoka H, Chiba K, Goto N and Kondo T: Effect of Wf-536, a novel ROCK inhibitor, against metastasis of B16 melanoma. Cancer Chemother Pharmacol 52(4): 319-324, 2003. PMID: 12783205. DOI: 10.1007/s00280003-0641-9

33 Díaz-Núñez M, Díez-Torre A, De Wever O, Andrade R, Arluzea J, Silió M and Aréchaga J: Histone deacetylase inhibitors induce invasion of human melanoma cells in vitro via differential regulation of $\mathrm{N}$-cadherin expression and RhoA activity. BMC Cancer 16(1): 667, 2016. PMID: 27549189. DOI: 10.1186/s12885-016-2693-3

34 Davies H, Bignell GR, Cox C, Stephens P, Edkins S, Clegg S, Teague J, Woffendin H, Garnett MJ, Bottomley W, Davis N, Dicks E, Ewing R, Floyd Y, Gray K, Hall S, Hawes R, Hughes J, Kosmidou V, Menzies A, Mould C, Parker A, Stevens C, Watt S, Hooper S, Wilson R, Jayatilake H, Gusterson BA, Cooper C, Shipley J, Hargrave D, Pritchard-Jones K, Maitland N, Chenevix-Trench G, Riggins GJ, Bigner DD, Palmieri G, Cossu A, Flanagan A, Nicholson A, Ho JWC, Leung SY, Yuen ST, Weber BL, Seigler HF, Darrow TL, Paterson H, Marais R, Marshall CJ, Wooster R, Stratton MR and Futreal PA: Mutations of the BRAF gene in human cancer. Nature 417(6892): 949-954, 2002. PMID: 12068308 . DOI: $10.1038 /$ nature00766

35 Smit MA, Maddalo G, Greig K, Raaijmakers LM, Possik PA, Breukelen B, Cappadona S, Heck AJ, Altelaar AM and Peeper DS: ROCK 1 is a potential combinatorial drug target for $B R A F$ mutant melanoma. Mol Syst Biol 10(12): 772, 2014. PMID: 25538140. DOI: $10.15252 / \mathrm{msb} .20145450$
36 Misek SA, Appleton KM, Dexheimer TS, Lisabeth EM, Lo RS, Larsen SD, Gallo KA and Neubig RR: Rho-mediated signaling promotes BRAF inhibitor resistance in de-differentiated melanoma cells. Oncogene 39(7): 1466-1483, 2020. PMID: 31659259. DOI: 10.1038/s41388-019-1074-1

37 Azreq M-A El, Kadiri M, Boisvert M, Pagé N, Tessier PA, Aoudjit F, El Azreq M-A, Kadiri M, Boisvert M, Pagé N, Tessier PA and Aoudjit F: Discoidin domain receptor 1 promotes Th17 cell migration by activating the RhoA/ROCK/MAPK/ERK signaling pathway. Oncotarget 7(29): 44975-44990, 2016. PMID: 27391444. DOI: 10.18632/oncotarget.10455

38 Lang P, Guizani L, Vitté-Mony I, Stancou R, Dorseuil O, Gacon $\mathrm{G}$ and Bertoglio J: ADP-ribosylation of the RAS-related, GTPbinding protein RhoA inhibits lymphocyte-mediated cytotoxicity. J Biol Chem 267(17): 11677-11680, 1992. PMID: 1601841.

39 Lou Z, Billadeau DD, Savoy DN, Schoon RA and Leibson PJ: A role for a RhoA/ROCK/LIM-kinase pathway in the regulation of cytotoxic lymphocytes. J Immunol 167(10): 5749-5757, 2001. PMID: 11698448. DOI: 10.4049/jimmunol.167.10.5749

40 Tharaux P-L, Bukoski RC, Rocha PN, Crowley SD, Ruiz P, Nataraj C, Howell DN, Kaibuchi K, Spurney RF and Coffman TM: Rho kinase promotes alloimmune responses by regulating the proliferation and structure of T-cells. J Immunol 171(1): 96105, 2003. PMID: 12816987. DOI: 10.4049/jimmunol.171.1.96

41 Mertsch S and Thanos S: Opposing signaling of ROCK1 and ROCK2 determines the switching of substrate specificity and the mode of migration of glioblastoma cells. Mol Neurobiol 49(2): 900-915, 2014. PMID: 24170433. DOI: 10.1007/s12035-0138568-6

42 Wang J, Liu X-H, Yang Z-J, Xie B and Zhong Y-S: The effect of ROCK-1 activity change on the adhesive and invasive ability of Y79 retinoblastoma cells. BMC Cancer 14: 89, 2014. PMID: 24528629. DOI: 10.1186/1471-2407-14-89

43 Kümper S, Mardakheh FK, McCarthy A, Yeo M, Stamp GW, Paul A, Worboys J, Sadok A, Jørgensen C, Guichard S, Marshall CJ, Li Z, Luo D, Shi F, Zheng Y, Bi F, Lyons J, Raynaud F, Eccles S, Workman P, Thompson N and Garrett M: Rhoassociated kinase (ROCK) function is essential for cell cycle progression, senescence and tumorigenesis. Elife 5: e12994, 2016. PMID: 26765561. DOI: 10.7554/eLife. 12203

44 Kamai T, Yamanishi T, Shirataki H, Takagi K, Asami H, Ito Y and Yoshida K-I: Overexpression of RhoA, RAC1, and CDC42 GTPases is associated with progression in testicular cancer. Clin Cancer Res 10(14): 4799-4805, 2004. PMID: 15269155. DOI: 10.1158/1078-0432.CCR-0436-03

45 Kamai T, Tsujii T, Arai K, Takagi K, Asami H, Ito Y and Oshima $\mathrm{H}$ : Significant association of Rho/ROCK pathway with invasion and metastasis of bladder cancer. Clin Cancer Res 9(7): 26322641, 2003. PMID: 12855641.

46 Gilkes DM, Xiang L, Lee SJ, Chaturvedi P, Hubbi ME, Wirtz D and Semenza GL: Hypoxia-inducible factors mediate coordinated RhoA-ROCK1 expression and signaling in breast cancer cells. Proc Natl Acad Sci USA 111(3): E384-393, 2014. PMID: 24324133. DOI: 10.1073/pnas.1321510111

Received February 11, 2020

Revised February 29, 2020

Accepted March 4, 2020 